\title{
Design criteria for multipulse operation of a multisection pulse line ion accelerator
}

\author{
D. Y. Lei* and Simon Yu \\ Department of Physics, The Chinese University of Hong Kong, Shatin, Hong Kong, People's Republic of China \\ and Lawrence Berkeley National Laboratory, Berkeley, California 94720, USA
}

(Received 21 August 2007; published 29 November 2007)

\begin{abstract}
We present a first study of the pulse line ion accelerator operating in a multipulse, multisection mode. The study is directed towards applications with long beam pulse in a long linac with a series of accelerating structures. We show that this operating mode is possible provided that the amplitude of the accelerating voltage waveform does not change over the entire pulse train and the operating frequencies of different sections are the same. A design criteria for the length and energy gain of a multisection linac is derived, and is applied to an example of a heavy ion linac injector.
\end{abstract}

DOI: 10.1103/PhysRevSTAB.10.110401

PACS numbers: 29.17.+w

\section{INTRODUCTION}

The pulse line ion accelerator (PLIA) [1] offers the possibility of a low-cost linac for the acceleration of nonrelativistic ions. It was originally motivated by the need to use moderate energy intense ion beams to heat matter to regimes of interest for studies of high energy density physics (HEDP) and warm dense matter [2-4]. In the design of PLIA, each accelerating structure consists of a long wire wound helically around an insulating vacuum tube and immersed in a dielectric medium [5]. This simple mechanical device acts as a traveling wave tube with a wave speed determined by the density (turns per unit length) of the helical winding, and by the dielectric constant and geometry of the insulating medium, i.e., the inductance and capacitance per unit length of a distributed transmission line. The accelerating field of the traveling wave comes from the spatial gradient of a time-varying voltage pulse. To be economically attractive, we want reasonably high gradient ( $\leq 2 \mathrm{MV} / \mathrm{m}$ ) at acceptably low voltages (say, $\sim 200 \mathrm{kV}$ ). Using a multiturn step-up input coupler, the input voltage would be in the tens of $\mathrm{kV}$ range [1]. The combined requirement on gradient and voltage implies that the optimal frequency is of the order of MHz. PLIA offers a uniquely attractive accelerator architecture in this regime [6].

For some applications, it would be desirable to accelerate a long train of ions at up to tens of microseconds. This would be possible if one could operate PLIA in a multipulse, multisection mode with a ringing circuit. In this paper, we consider the beam dynamics of such an accelerator, and derive a set of criteria for designing an accelerator with multiple sections, using PLIA structures.

\section{MULTIPULSE BEAM DYNAMICS}

Consider a train of short ion bunches (of charge $q$ and mass $m$ ), separated by time period $T$, entering a PLIA

\footnotetext{
*dylei@phy.cuhk.edu.hk
}

section of length $L$ at speed $v_{o}$. In this paper, we consider only the case of constant wave speed without tapering. Future optimizations for specific applications may generalize the present study to tapered helices. We approximate each short bunch by a single macroparticle whose arrival time at a position $z$ is given by $t^{i}(z)$, with $i$ denoting the $i$ th particle bunch. In the wave frame, the time of the particle relative to the wave is given by

$$
\tau^{i}(z)-\tau_{o}^{i}=\left[t^{i}(z)-t_{o}^{i}\right]-\frac{z-z_{o}}{v_{w}},
$$

where $v_{w}$ is the wave speed, and the subscript $o$ denotes a quantity at PLIA entrance.

\section{A. Constant frequency operation}

The simplest way to operate a PLIA in a multipulse mode is to demand that the frequency of the ringing circuit $f$ be synchronized with that of the entering particle train. The period is $T=1 / f$. The phase of all particle bunches relative to the wave is identical at entry

$$
\tau_{o}^{i}=\tau_{o} \quad \text { for all } i .
$$

As the particles move down the PLIA section, their motion in the wave frame is the same:

$$
\tau^{i}(z)=\tau(z) \quad \text { for all } i,
$$

i.e. all particles may "slosh" back and forth in the wave frame, but at any given position $z$, the phase of all particles relative to the wave is the same. It follows that the frequency at any given position $z$ remains unchanged, and the exit frequency is the same as the entrance frequency. We will henceforth drop the superscript $i$.

As the ion train goes from one section to the next, acceleration will be uniform throughout the entire train as long as the frequency remains unchanged. The wave speed $v_{w}$ and the accelerating gradient $G$ may vary of course from section to section.

If the frequency is fixed, and the speed increases as the beam is accelerated, the spacing between ion bunches as 
well as the wavelength of successive sections $L_{w}=v_{w} T$ will increase. The requirement of fixed frequency places constraints on both the beam and the accelerating voltage pulse.

For the beam, it implies that the duration of the entire train will remain fixed, and the length of the entire train will increase as the beam is accelerated. Whatever bunching and additional manipulations of the macropulse one wishes to do will have to be performed postacceleration.

For the voltage pulse, since the gradient is proportional to $\tilde{V} / L_{w}$, where $\tilde{V}$ is the peak voltage, either the gradient has to drop or the peak voltage has to continuously increase. This feature places some constraints on how many PLIA sections and how much energy gain could be achieved in a PLIA-based linac.

This constraint can be relaxed if one observes that the requirement $\tau^{i}(z)=\tau(z)$ can also be met if the frequency is doubled. With frequency doubling, every other rf bucket is filled. If one wishes to design a long accelerator with constant gradient, it is possible by doubling the frequency at discrete intervals down the accelerator. For the application considered in the next section, we do not need to invoke this "trick".

\section{B. Beam trapping and ion slosh time}

The concepts of beam trapping and ion slosh time are essential to the design of PLIA-based accelerators. We begin by considering an idealized voltage pulse as shown in Fig. 1. At the input end of the helix a pulse power source creates a sawtooth shape waveform. A similar model was considered by Briggs [1]. In the linear ramp region, the voltage goes from $-\tilde{V}$ to $+\tilde{V}$ in a time scale of $\bar{\tau}$ with a length scale of $\bar{\tau} v_{w}$.

During the traversal of the beam through the PLIA structure, the ion bunch will slosh back and forth relative to the wave. However, in order for the PLIA to be an effective accelerating structure, the ion must always stay within the region $\bar{\tau}$. As soon as it falls out of this region, deceleration occurs, and the beam is "detrapped."

The ion speed at entry $v_{o}$ should be lower than the wave speed $v_{w}$. The ion speed continues to increase in the structure, until it reaches $v_{f}$ at exit, which is generally

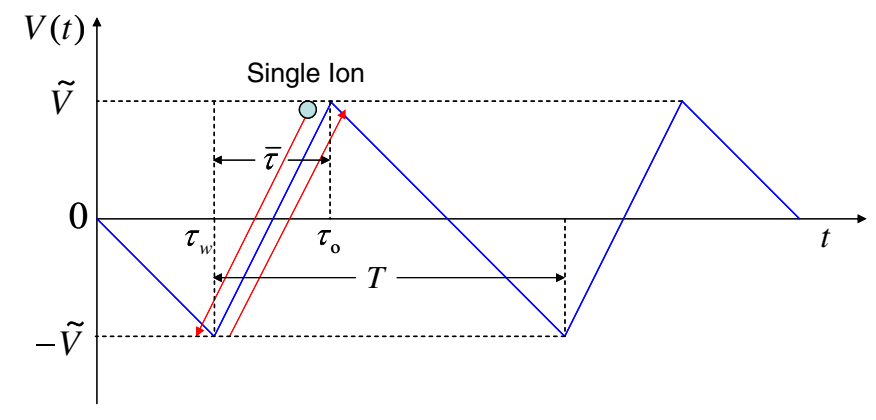

FIG. 1. (Color) Voltage waveform at the helix input $z=z_{o}$. somewhat higher than $v_{w}$. As long as $v$ is less than $v_{w}$, the beam moves backward in the wave frame, and $\tau$ would continue to decrease until it reaches a critical point when $v=v_{w}$. As $v$ continues to increase past this point, the beam begins to move forward in the wave frame, and $\tau$ would also increase. The most efficient use of the accelerator is to let the beam enter at $\tau_{o}$. We would arrange for the critical point where $v=v_{w}$ to be at $\tau_{w}$, and then allow the beam to exit when $\tau$ returns to its point of origin in the wave frame, i.e.

$$
\tau(L)=\tau_{o}
$$

The excursion of the beam within the wave frame lies within the region

$$
\bar{\tau} \equiv \tau_{w}-\tau_{o}
$$

The significance of $\bar{\tau}$ is that it places constraints on the length of the PLIA section and the energy gain. The longer the PLIA section and the larger the energy gain, the more the ion bunch will slosh, and the larger the $\bar{\tau}$. A bound on $\bar{\tau}$ is also a bound on energy gain per section. We now proceed to calculate this relationship in the constant acceleration approximation,

$$
a=\frac{q}{m} G
$$

where $a$ is the acceleration, and $G=|d V / d Z|=2 \tilde{V} / \bar{\tau} v_{w}$ is the longitudinal electric field gradient.

\section{Dimensionless parameter $\lambda$ and energy gain per section}

We note that the initial conditions of particles relative to the wave can be chosen by controlling the input time and position of particles into the PLIA section. In order to further simplify our calculations, we choose $z_{o}=0, t_{o}=$ $0, \tau_{o}=0$. Based on such a choice, we can rewrite the phase of particle relative to the wave as follows:

$$
\tau(z)=t(z)-\frac{z}{v_{w}} .
$$

The equations of motion are as follows:

$$
v \frac{d v}{d z}=a \quad \frac{d t}{d z}=\frac{1}{v} .
$$

By solving the above equations together with the initial conditions, $v$ and $\tau$ are given by

$$
v^{2}-v_{o}^{2}=2 a z \quad \tau=\frac{v-v_{o}}{a}-\frac{z}{v_{w}},
$$

where $v_{o}$ is the initial velocity of the particle at the input end. By solving Eq. (9) at the critical point where $v=v_{w}$, the "slosh time" $\bar{\tau}$ is given by

$$
\bar{\tau}=t_{w}-\frac{z_{w}}{v_{w}}=\frac{v_{w}}{2 a}+\frac{v_{o}^{2}}{2 a v_{w}}-\frac{v_{o}}{a},
$$


where $t_{w}$ and $z_{w}$ are the arrival time and arrival position of the particle at the critical point. Again by solving Eq. (9) at exit where $\tau(L)=0$, the wave speed is given by

$$
v_{w}=\frac{v_{f}+v_{o}}{2} .
$$

We then obtain a simple relation between a dimensionless parameter $\lambda$ and a dimensionless function $h\left(\frac{v_{f}}{v_{o}}\right)$,

$$
\lambda=h\left(\frac{v_{f}}{v_{o}}\right)
$$

where

$$
h(r)=\frac{(r-1)^{2}}{4 r(r+1)}
$$

and

$$
\lambda=\frac{q}{m}\left(\frac{\bar{\tau} G}{v_{f}}\right) .
$$

Figure 2 shows a plot of $h(r)$ as a function of $r$. We note that $\lambda$ is a parameter specified externally by the voltage waveform (the slosh time $\bar{\tau}$ ), the desired gradient $G$, and the final velocity $v_{f}$ one wishes to achieve. Equation (12) then tells us the initial velocity of that section, from which of course we obtain the energy gain $\Delta E=\frac{1}{2} m v_{f}^{2}-\frac{1}{2} m v_{o}^{2}$. Equations (12)-(14) allow us to relate the energy gain in a given section to the slosh time and the accelerating gradient. Since we know the energy gain and the gradient, the section length $L$ is trivially derived. This equation gives an upper limit for the energy gain of a single PLIA section.

It is also useful to rewrite the dimensionless parameter $\lambda$ as the following form:

$$
\lambda=\frac{q}{m}\left(\frac{\bar{\tau} G v_{w}}{v_{f} v_{w}}\right)=\frac{4 q \tilde{V}}{m v_{f}^{2}\left(1+v_{o} / v_{f}\right)} .
$$

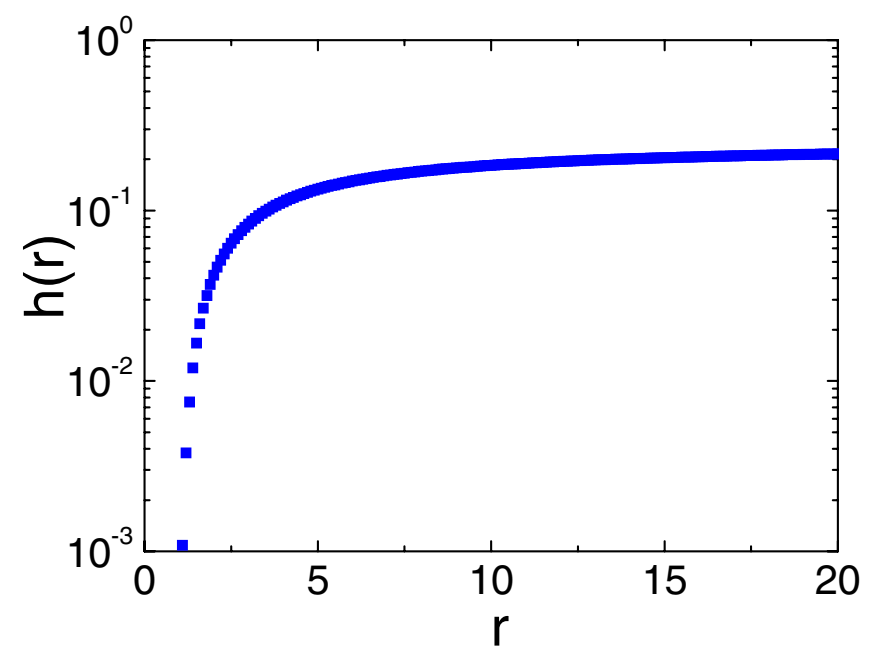

FIG. 2. (Color) Sketch of dimensionless function $h(r)$ as a function of $r$.
This equation shows that, for the special sawtooth example that we have considered, the maximum energy gain depends only on the peak voltage.

In the above derivation, we have not considered the gain or loss of energy introduced as the ion bunch passes through the wave launching region (the entrance) and the resistive termination region (the exit). The reason is that, for the case considered, the phase at the entrance is the same as the phase at the exit, and the small energy gain at entrance is canceled exactly by the energy loss at exit. In addition, since we have approximated each short bunch by a single macroparticle, the energy spread can be ignored. For finite pulse lengths, the energy spread effects must be carefully evaluated.

\section{An approximation for the sinusoid and more general waveforms}

For waveforms that are different from the sawtooth model used in the previous calculations, the relation would be somewhat more complicated. In general, the "slosh time" $\bar{\tau}$ can be varied from section to section, but it must always be less than the period $T$. It is bounded by the frequency and waveform of the voltage pulse, and Eq. (10) can still be applied to the region where the gradient is approximately constant.

As an example, if the voltage waveform is purely sinusoidal, we note that the gradient is approximately linear between $\pm 30^{\circ}$, i.e. $\bar{\tau} \omega \approx 1(\omega=2 \pi / T)$. When the particle resides in this region, the gradient can be expressed as $G \approx \tilde{V} \omega / v_{w}$, where $\tilde{V}$ is the peak voltage of the sinusoidal wave. We can rewrite Eq. (12) as

$$
\tilde{\lambda}=\tilde{h}\left(\frac{v_{f}}{v_{o}}\right),
$$

where

$$
\tilde{h}\left(\frac{v_{f}}{v_{o}}\right)=\frac{1}{2}\left(1+\frac{v_{o}}{v_{f}}\right) h\left(\frac{v_{f}}{v_{o}}\right)
$$

and

$$
\tilde{\lambda}=(\bar{\tau} \omega)\left(\frac{q \tilde{V}}{2 E_{f}}\right) .
$$

Equation (18) allows us to write the dimensionless parameter in terms of the peak voltage $\tilde{V}$ and the "sloshing phase" $(\bar{\tau} \omega)$.

It is important to show that the PLIA can work in principle for a long linac. In the high energy limit, where the energy gain is a small fraction of the total beam energy, $\lambda \approx \tilde{\lambda}$ and $h \approx \tilde{h}$, Eqs. (16)-(18) reduce to the simple form:

$$
\Delta E=4 E_{o}^{1 / 2}[q \tilde{V}(\omega \bar{\tau})]^{1 / 2} \quad\left(\text { limit of } \frac{\Delta E}{E_{o}} \ll 1\right) .
$$

Equation (19) allows that we can make substantial gains in 


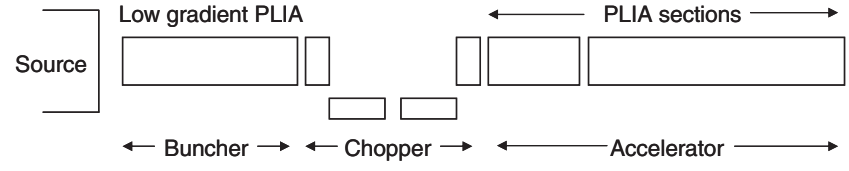

FIG. 3. A schematic of a PLIA-based system with buncher and chopper. The ion buncher consists of a low gradient PLIA, and energy selection and beam chopping is accomplished by a magnetic chicane with four quadrupoles.

energy per section without resorting to very high peak voltages.

\section{DESIGN EXAMPLE: THE FRONT END OF A HIGH CURRENT HEAVY ION INJECTOR}

One possible application of the concept derived is in a high current injector for a storage ring where PLIA may be a substitute for the radiofrequency quadrupole section of the linac. The parameters relevant to the machine under consideration are as follows [7]:

$$
\begin{gathered}
{ }_{92}^{238} U^{28+}, \quad E_{o}=7 \mathrm{kV} / u, \quad E_{f}=500 \mathrm{kV} / u, \\
20 \mu \text { s at } 2 \mathrm{~mA}, \quad 10 \mathrm{~Hz} .
\end{gathered}
$$

A schematic of a PLIA-based system is shown in Fig. 3.

We make the following observations regarding this application: (i) The total required voltage to reach the final energy is 4.25 MV. (ii) The average current is low, and space charge effects are less severe than the typical HIFVNL HEDP application [8]. (iii) The incoming energy is almost identical to the PLIA experiment at NDCX, and the first bunching section proposed in this design is very similar to Roy et al. [9].

The buncher takes a DC beam from the source and creates a beam bunched at the desired operating frequency with large energy oscillations in the longitudinal phase space. The chopper is an energy filter which eliminates the unwanted particles. This buncher-chopper combination produces a train of short bunches at the desired frequency. Detailed calculation of the buncher-chopper dynamics is outside of the scope of this paper.

Since we do not have a full calculation including the buncher and the chopper, we will present a calculation that takes bunched beams from the final energy of $500 \mathrm{kV} / u$ all the way down to the initial energy of $7 \mathrm{kV} / u$. This example turns out to consist of three sections. It is likely that the lowest energy section can be replaced by the buncherchopper combination.

To design the PLIA-based accelerator, we start from the output end where $E_{f} / q=4.25 \mathrm{MV}$ and $v_{f}=9.79 \times$ $10^{6} \mathrm{~m} / \mathrm{s}$ (this final velocity is consistent with $E_{f}=$ $500 \mathrm{kV} / u$ ). We would like to keep the system compact, and yet maintain a safe break-down-free system. We therefore choose a conservative gradient of less than $2.0 \mathrm{MV} / \mathrm{m}$. (For the purpose of this design, we will assume that a solution to the anomalous flashover observed in [9] has been found, and that the more conventional insulator gradient of $2.0 \mathrm{MV} / \mathrm{m}$ can be achieved. In fact, Waldron et al. [10] have made significant advances towards the understanding of the problem.) The full accelerator length is less than $4 \mathrm{~m}$.

If the frequency of various sections is fixed, then the wavelength is longest at the high energy sections in the downstream end. If the gradient is fixed, then the peak voltage has to vary, with the highest voltage at the downstream end. We will start our design from that end. We will limit the peak voltage to $200 \mathrm{kV}$. Using a multiturn input coupler for voltage step-up, the input voltage is in the range of tens of $\mathrm{kV}$ and the feedthrough should be straightforward.

Assuming a sinusoidal wave, we choose $\bar{\tau} \omega=1.1$. This is a relatively conservative choice of sloshing phase, but allows us to use the constant gradient formulas derived in Sec. II. With this rather conservative choice, we are also providing room for beam trapping when the present single macroparticle analysis is extended to the case of more realistic beams with finite lengths. With these choices and the required output energy, $\tilde{\lambda}$ is specified, and Eq. (16) can be solved to give $\Delta E=E_{f}\left[1-\left(v_{o} / v_{f}\right)^{2}\right]=$ $351.5 \times 10^{3} \mathrm{~V} / u$, Hence $E_{o}=E_{f}-\Delta E=148.5 \times$ $10^{3} \mathrm{~V} / u$ and $v_{w}=7.6 \times 10^{6} \mathrm{~m} / \mathrm{s}$. Applying this result to Eq. (14), assuming a gradient of $1.4 \mathrm{MV} / \mathrm{m}$, we obtain the "slosh time" $\bar{\tau}=20.8 \mathrm{~ns}$ and the design frequency $f=\omega / 2 \pi=9.15 \times 10^{6} \mathrm{~Hz}$. The length of this section is $2.13 \mathrm{~m}$.

We note that the frequency is now fixed for the lower energy PLIA sections. We can no longer specify gradient and peak voltage at the same time. We will choose the gradient, and allow the peak voltage to be a derived quantity. The input energy to the last (high energy) section is now viewed as the exit energy of the penultimate section. If we choose $G$ to be $1.8 \mathrm{MV} / \mathrm{m}$, and again $\bar{\tau} \omega \approx 1.1$, Eq. (16) can be used to obtain $\Delta E=130.6 \times 10^{3} \mathrm{~V} / u$,

TABLE I. The detailed design parameters of the multipulse PLIA for IMP in Lanzhou.

\begin{tabular}{lccccccc}
\hline \hline Section & $E_{f}(\mathrm{kV} / u)$ & $E_{o}(\mathrm{kV} / u)$ & $L(\mathrm{~m})$ & $f(\mathrm{MHz})$ & $v_{w}\left(10^{6} \mathrm{~m} / \mathrm{s}\right)$ & $G(\mathrm{MV} / \mathrm{m})$ & $\tilde{V}(\mathrm{kV})$ \\
\hline I & 17.9 & 7.5 & 0.59 & 9.15 & 1.5 & 0.15 & 4.3 \\
II & 148.5 & 17.9 & 0.79 & 9.15 & 3.6 & 1.8 & 122 \\
III & 500 & 148.5 & 2.13 & 9.15 & 7.6 & 1.4 & 200 \\
\hline \hline
\end{tabular}


$E_{o}=17.9 \times 10^{3} \mathrm{~V} / u, \quad v_{w}=3.6 \times 10^{6} \mathrm{~m} / \mathrm{s}, \quad L=$ $238 \Delta E / 28 G=0.79 \mathrm{~m}$.

In the similar way, we can get the parameters of the initial section with an input initial energy $E_{o}=$ $7.5 \times 10^{3} \mathrm{~V} / u$, which is very close to the required initial energy $E_{o}=7.0 \times 10^{3} \mathrm{~V} / u$. In the design of the low energy section, we chose a very low gradient, so that the mechanical length of the section is not too short. The parameters of all three sections are shown in Table I.

Hence a three-section PLIA appears adequate for the purpose of this particular application.

\section{CONCLUSION}

In this paper, we show that multipulse operation of a pulse line ion accelerator through a long linac with multisections is indeed possible. This is important for a class of applications where high average current is essential. Thus, for example, PLIA now offers the possibility for a different high current injector for heavy ion accumulator rings or the front end of a high current injector for protons. PLIA has unique advantages in terms of simplicity and cost of the accelerating structures, and is also practically suited to low frequency (few to few tenths of $\mathrm{MHz}$ ). The issues of power source and focusing magnets must be included when we evaluate the cost competitiveness of PLIA versus other more conventional architecture, for example, the radiofrequency quadrupole. These two major cost items are likely to lead to a reoptimization of the system parameters. Multipulse operation would extend its utility significantly. The most straightforward operation mode considered in this paper limits the pulse train to the same energy for every microbunch at a given position. The pulse train duration (in time) remains constant with acceleration, and the pulse length (in space) increases. Any required beam bunching manipulations will have to be performed postacceleration.

In addition to the constant pulse duration requirement, the multipulse PLIA operation also places a constraint on the frequency of successive sections. Constant frequency for all sections would imply lower gradient or higher peak voltage for the high energy end of a PLIA-based linac. This limitation could be alleviated somewhat by noting that higher harmonics of the fundamental frequency are also allowed. In addition, the reduction in gradient or increase in peak voltage with increasing energy is sufficiently slow that the constraint on a multisection linac may not be severe in practical applications.

We derive a design equation that allows us to determine the energy gain and section length for successive accelerating structures in a PLIA-based linac. This design equation is applied to the example of a high current injector for a heavy ion accumulator ring. We show that a compact three-section PLIA may be an alternative to a more conventional radiofrequency linac section.

The analysis in this paper is based on straightforward kinematic considerations. Issues of space charge, beam loading etc. are not considered. For various applications, the results from this paper may provide a zeroth order design guide for more detailed simulations.

\section{ACKNOWLEDGMENTS}

We would like to thank Dr. B. G. Logan of the Lawrence Berkeley National Laboratory for useful discussions and valuable suggestions. We would also like to thank Professor Hongwei Zhao of the Institute of Modern Physics at the Heavy ion Research Facility of Lanzhou for kindly providing us with the parameters they are considering for their HIRSF-CSR injector.

[1] R. J. Briggs, Phys. Rev. ST Accel. Beams 9, 060401 (2006).

[2] L. Grisham, Phys. Plasmas 11, 5727 (2004).

[3] A. Friedman et al., in Proceedings of the 2005 Particle Accelerator Conference, Knoxville, Tennessee, 2005, p. 339.

[4] J. J. Barnard et al., in Proceedings of the 2005 Particle Accelerator Conference, Knoxville, Tennessee, 2005, p. 2568.

[5] R.J. Briggs, in Proceedings of the 2005 Particle Accelerator Conference, Knoxville, Tennessee, 2005, p. 440.

[6] J. E. Coleman et al., Nucl. Instrum. Methods Phys. Res., Sect. A 577, 197 (2007).

[7] Hongwei Zhao (private communication).

[8] P. K. Roy et al., Phys. Rev. Lett. 95, 234801 (2005).

[9] P. K. Roy et al., Phys. Rev. ST Accel. Beams 9, 070402 (2006).

[10] W.L. Waldron et al., Proceedings of the 2007 Particle Accelerator Conference, Albuquerque, 2007, p. 852. 